\title{
Optimal Control for an Irrigation Planning Problem: characterisation of solution and validation of the numerical results
}

\author{
Sofia O. Lopes $^{13}$, Fernando A.C.C. Fontes ${ }^{2}$, Rui M.S. Pereira ${ }^{14}$, M. Rosário de \\ Pinho $^{2}$, and C. Ribeiro ${ }^{1}$ \\ 1 CMAT and Departamento de Matemática e Aplicações, Universidade do Minho, \\ Guimarães, Portugal \\ 2 ISR-Porto, Faculdade de Engenharia, Universidade do Porto, Porto, Portugal \\ ${ }^{3}$ Collaborator with ISR-Porto, Universidade do Porto \\ 4 Collaborator with Centre of Physics, Universidade do Minho
}

\begin{abstract}
In a previous study, the authors developed the planning of the water used in the irrigation systems of a given farmland in order to ensure that the field cultivation is in a good state of preservation. This planning was modelled and tackled as an optimal control problem: minimize the water flow (control) so that the extent water amount in the soil (trajectory) fulfils the cultivation water requirements. In this paper, we characterize the solution of our problem guaranteeing the existence of the solution and applying the necessary and sufficient conditions of optimality. We validate the numerical results obtained previously, comparing the analytical and numerical solutions.
\end{abstract}

\section{Introduction}

The climate system and the ecosystems are under accelerated change while human cultures, economic activities and national interactions are undergoing dramatic and, sometimes, exponential changes. The rapid increase of the world population leads to a soaring demand of water. Agriculture exerts pressure on the environment, especially on water. Thus, appropriate water management throughout the irrigation processes is needed.

A model to optimize the water use in the irrigation of a farm field via optimal control (water flow) that takes into account the evapotranspiration, rainfall, losses by infiltration and runoff was developed in [9]. There the problem is introduced, and a solution obtained for the "Yearly Planning" problem considering different weather scenarios with the help of the so called "precipitation factor" that is multiplied by the rainfall monthly average. In [10], the authors present the "Initial Planning Problem" for rainfall: this includes an extra term taking into account the rainfall in the previous time period (this rainfall model was statistically proven to be significant) where a comparison between this new model and the solution knowing the rainfall a priori was shown. 
In this paper, we show that the solution of Initial Planning Problem exists and we characterize it using necessary and sufficient conditions of optimality. We also compare the results obtained analytically and numerically.

This paper is organized as follows. In section 2 we present a model for the planning of irrigation based on the hydrologic balance equation. In section 3 we show the analytical study of the solution. In section 4 , we present a numerically implementation of the problem and we show that the numerical results agree with the results obtained analytically.

\section{A Model for Planning the Irrigation}

We consider a simple model for the planning of the water used in the irrigation of farm fields, based on the hydrlogic balance. This means that, the variation of water in the soil is given by

$$
\dot{x}=u+\mathbf{r f a l l}-\mathbf{e v t p}-\mathbf{r n o f f}
$$

where $x$ is the water in the soil, $u$ is the amount of water flow introduced in the soil via its irrigation system, the rfall is rainfall, the evtp is the evaporation of the soil and the transpiration of the crop and rnoff are the losses of water due to the runoff and deep infiltration.

Having in mind the postulate of Horton's equation, infiltration decreases exponentially with time [4]. The dynamical equation can be written as

$$
\dot{x}(t)=l(t)-w(t) x(t)
$$

where $l(t)=u(t)+\mathbf{r f a l l}(t)-\operatorname{evt} \mathbf{p}(t)$.

From (1) and (2), one may say $\mathbf{r n o f f}=\beta x$, where $\beta$ is a parameter that depends on the type of soil.

From now on, we consider the following dynamic equation

$$
\dot{x}=u+g(t)-\beta x,
$$

where $g(t)=\mathbf{r f a l l}(t)-\operatorname{evt} \mathbf{p}(t)$ and $\beta$ is the percentage of losses of water due to the runoff and deep infiltration.

The dynamic equation (3) is solved over the time interval $[0, T]$ subject to the initial condition $x(0)=x_{0}$. The variable of state $x$ is subject to the inequality constraint $x \geq x_{\min }$, where $x_{\min }$ is the hydrological need of the crop (according to [8]). The control variable $u$ belongs to the interval $[0, M]$, where $M$ is the maximum flow of water that comes from tap. 
The optimal control problem (OCP) formulation is then:

$$
\begin{aligned}
& \min \int_{0}^{T} u(t) d t \\
& \text { subject to: } \\
& \dot{x}(t)=u+g(t)-\beta x \text { a.e. } t \in[0, T] \\
& x_{\min }-x \leq 0 \quad \forall t \in[0, T] \\
& u(t) \in[0, M] \\
& x(0)=x_{0} .
\end{aligned}
$$

A detailed description of this models is given in [10].

\section{Analysis of the Solution}

In order to characterize the solution of our problem, we prove the existence of solution and we apply the necessary and sufficient conditions of optimality.

Throught, the function $H$ represents the pseudo-Hamilton function:

$$
H(t, x, p, u, \lambda)=p(u+g(t)-\beta x)-\lambda u
$$

where $p$ and $\lambda$ are Lagrangian multipliers.

\subsection{Existence of Solution}

Existence of solution was introduced by Tonelli (1915) when he proposed the first theorem of existence of solution for calculus of variations problems. Even today, Tonelli's theorem remains the central existence theorem for dynamic problems, although the hypotheses of the theorem can be relaxed, see, for example, [12]. In this section, we apply the theorem 5.4.4. in [2] to guarantee the existence of solution for our OCP. Let us verify the conditions of this theorem, see theorem (in Appendix)):

* The dynamic function and cost function are differentiable in the state and control variable and the control belongs to the interval $[0, M]$.

* The condition i) of theorem 5.4.4. is satisfied, since the set

$$
\{[u+g(t)-\beta x, u+\delta]: u \in[0, M] \text { and } \delta \geq 0\}
$$

is convex. Indeed, this set is the epigraph of a convex function.

* For the condition ii): take $\sigma(t)=\beta, \rho(t)=0$ and $\phi(t, p)=|p-1| M+|p||g(t)|$. Then we have

$$
p(u+g(t)-\beta x)-u \leq|p-1| M+|p||g(t)|+\beta|p||x| .
$$

* The set $C_{0}=\left\{x_{0}\right\}$ is compact, so the condition iii) is satisfied.

The assumptions of the theorem 5.4.4. in [2] are verified and we conclude that there exists an admissible solution to our OCP. 


\subsection{Necessary and Sufficient conditions of optimality}

In this section, we start by verifying that our problem satisfies the constraint qualification that allows to write the Maximum Principle (MP) in the normal form: the multiplier associated to the objective function $\lambda$ is not zero (see [3] and [7] for discussion of normal forms of the MP for optimal control problems with state constraints). We finish this section applying the MP in the normal form and we verify that MP conditions are also sufficient conditions.

\section{Step 1: Verify the normality}

In Rampazzo and Vinter [7], the MP can be written with $\lambda=1$, if there exists a continuous feedback $u=\eta(t, \xi)$ such that

$$
\frac{d h(\xi(t))}{d t}=h_{t}(t, \xi)+h_{x}(t, \xi) \cdot f(t, \xi, \eta(t, \xi))<-\gamma^{\prime}
$$

for some positive $\gamma^{\prime}$, whenever $(t, \xi)$ is close to the graph of the optimal trajectory, $\bar{x}(\cdot)$, and $\xi$ is near to the state constraint boundary. There should exist a control (flow of water provided by the irrigation systems) pulling the state variable away from the state constraint boundary (this guarantees that the crop survives).

In our problem $h(x)=x_{\min }-x$ and, from (6), we write

$$
\frac{d h(\xi(t))}{d t}=h_{x}(\xi(t)) \cdot f(t, \xi,, \eta(t, \xi))=-(\eta(t, \xi)+\triangle(t, \xi)) \leq-\gamma^{\prime},
$$

where $\triangle(t, \xi)=g(t)-\beta \xi$. For a $\xi$ on a neighbourhood of $\bar{x}$, we can always choose $\eta$ sufficiently large so that the equation (7) is satisfied, as long as $M \geq \beta \bar{x}(t)-g(t)$, a condition we can impose with loss of generality.

Thus the inward pointing condition (7) is satisfied and normality follows.

\section{Step 2: Application of the Maximum Principle.}

A known form of the normal MP for smooth problems with state constraint is:

Let $(\bar{x}, \bar{u})$ be a minimizer for (OCP), then there exists an absolutely continuous function $p$ and $\mu \in C^{*}(0,1)$ such that,

$$
\begin{aligned}
& -\dot{p}(t)=H_{x}(t, \bar{x}(t), q(t), \bar{u}(t), 1) \\
& H(t, \bar{x}(t), q(t), \bar{u}(t), 1)=\max _{v \in[0, M]} H(t, \bar{x}(t), q(t), v, 1) \text { a.e.; } \\
& \operatorname{supp}\{\mu\} \subset\{t \in[0, T]: h(\bar{x}(t))=0\} \\
& q(T)=0,
\end{aligned}
$$

where $q(t)$ is defined as follows,

$$
q(t)=\left\{\begin{array}{l}
p(t)-\int_{[0, t)} \mu(d s), \quad t \in[0, T) \\
p(T)-\int_{[0, T]} \mu(d s), \quad t=T .
\end{array}\right.
$$


Applying theses conditions to our problem, we have:

$$
\begin{aligned}
& \dot{p}(t)=\beta q(t) \\
& q(t)(\bar{u}(t)-u(t))-(\bar{u}(t)-u(t)) \geq 0 \\
& \operatorname{supp}\{\mu\} \subset\left\{t \in[0, T]: \bar{x}(t)=x_{\min }\right\} \\
& q(T)=0 .
\end{aligned}
$$

\section{Step 3: Application of the first order sufficient conditions}

We can apply sufficient conditions to our problem. Sufficiente conditions given by corollary of theorem 5.4.2 from [2] are validated under assumptions verified by the data of our problem, i.e., (we refer the reader to see corollary 1 in the appendix):

* The dynamic function and cost function are differentiable in the state and control variable and the control belongs to the interval $[0, M]$.

* The conditions (8) of the MP are satisfied.

* For each $t$ the function $(x, u) \rightarrow p(t)(u+g(t)-\beta x)-u-\psi_{[0, M]}(u)$ is concave.

Thus, the necessary conditions of optimality of our problem are also sufficient conditions.

\subsection{Characterization of solution}

Now, we characterize the optimal solution for (OCP) studying the Weierstrass condition of the MP for $\bar{u}=0, \bar{u}=M$ and $\bar{u} \in] 0, M[$.

If $\bar{u}=0$, we have that for all $u(t) \in[0, M]$,

$$
q(t) u(t)-u(t) \leq 0 \Leftrightarrow q(t) \leq 1 .
$$

If $\bar{u}=M$, we have that for all $u(t) \in[0, M]$,

$$
(q(t)-1)(M-u) \geq 0 \Leftrightarrow q(t) \geq 1 .
$$

In the remaining case (i.e. $\bar{u} \in] 0, M[$ ), we have:

$$
\begin{aligned}
& q(t)(\bar{u}(t)-u(t))-(\bar{u}(t)-u(t)) \geq 0 \Leftrightarrow \\
& (q(t)-1)(\bar{u}(t)-u(t)) \geq 0 \Leftrightarrow q(t)=1 .
\end{aligned}
$$

Next we shall use all this information to validate the numerical solution (already presented in [11]) of our problem. 


\section{Numerical Implementation and Results}

The numerical simulations of our problem requires its discretization. Its discrete formulation is as follows:

$$
\begin{aligned}
& \min \delta \sum_{i=1}^{N} u_{i} \\
& \text { such that: } \\
& \begin{array}{ll}
x_{i+1}=x_{i}+\delta f\left(t_{i}, x_{i}, u_{i}\right), \text { a.e. } i=1, \ldots, N, \\
x_{1}=x_{0} & i=1, \ldots, N, \\
x_{i} \geq x_{\min }, & \text { a.e. } i=1, \ldots, N, \\
u_{i} \in[0, M], &
\end{array}
\end{aligned}
$$

where $x=\left(x_{1}, \ldots, x_{N}\right)$ is the trajectory, $u=\left(u_{1}, \ldots, u_{N-1}\right)$ is the control, $f$ is the hydrological balance function, $x_{\min }$ is the hydrological need of the crop, $x_{0}$ is an initial state, $\delta$ is the time step discretization, and $N=T / \delta$. In the dynamic equation, $f$ is defined by

$$
f\left(t_{i}, x_{i}, u_{i}\right)=u_{i}+\operatorname{rainfall}\left(t_{i}\right)-\operatorname{evapotranspiration}\left(t_{i}\right)-\operatorname{losses}\left(x_{i}\right),
$$

where the evapotranspiration takes in account the evaporation of the soil and the transpiration of the crop and the losses are the losses of water due to the runoff and deep infiltration. The Rainfall model [10] is based on a linear combination of average monthly rainfall from the last 10 years and the amount of rainfall in the previous month. The evapotranspiration model is described as the crop coefficient (in our case potatoes) multiplied by the reference value of evapotranspiration in Lisbon, given by Pennman-Monteith methodology, (see [5]). The losses are model as $15 \%$ of the water in the soil, based on Horton's equations. A more detailed description of these models can be seen in [10].

The state constraint $\left(x_{i} \geq x_{\min }\right)$ translates the fact that the plants needs a minimum amount flow of water to survive.

We consider a field of potatoes in the region of Lisbon, Portugal. Based on data from [6] we consider:

$$
\begin{array}{ll}
x_{\min }=0.56 / 12 \mathrm{~m}^{3} / \text { month } & T=12 \\
x_{0}=4 x_{\min } \mathrm{m}^{3} / \mathrm{month} & \beta=15 \% \\
M=1 \mathrm{~m}^{3} / \mathrm{month} . &
\end{array}
$$

To obtain the numerical solution for the optimal control problem we have approximate the problem by a sequence of finite dimensional nonlinear programming problems, (see [1]).

To implement this optimization problem, we use fmincon function of MatLab with the algorithm "active set", by default.

The code produces results that are according to what is expected for this region [8].

Next, we plot the numerical solution and the expected multipliers. 


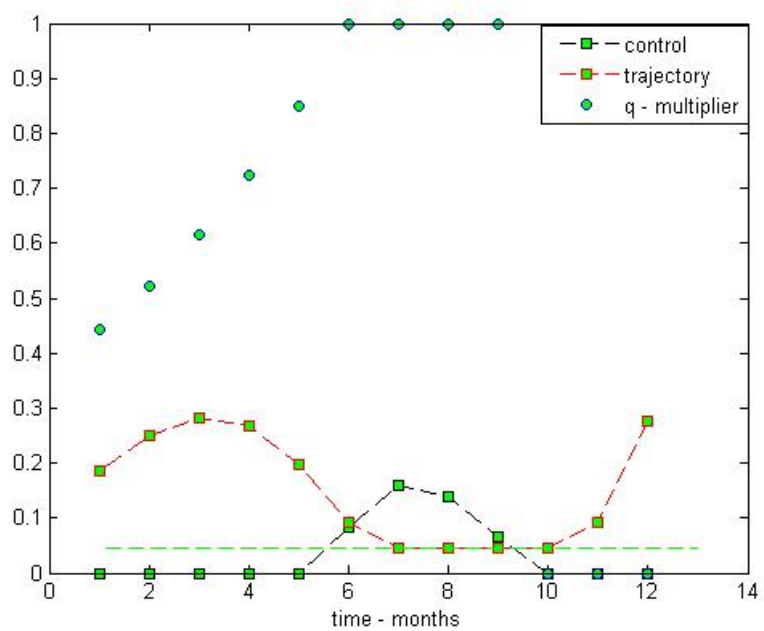

Note that the green line represents the hydrological need of the crop.

We can observe that:

$$
q(t) \leq 1 \text { if } \bar{u}=0, \quad q(t)=1 \text { if } \bar{u} \in] 0, M[
$$

and since $\bar{u}$ is never equal to $1, q$ is never great than 1 , as expected from section 3.3. From here, we can say that although the analytical explicit solution was not obtained, the numerical solution fulfils the necessary optimality conditions.

Our numerical findings suggest that the trajectory has a "boundary interval" $\left[t_{\text {in }}, t_{\text {out }}\right]$, with $t_{\text {in }}>0$ and $t_{\text {out }}<12$ (i.e. $\bar{x}(t)=x_{\min }$ for all $t \in\left[t_{\text {in }}, t_{\text {out }}\right]$ and $\bar{x}(t) \neq x_{\min }$ for $\left.t \notin\left[t_{\text {in }}, t_{\text {out }}\right]\right)$ and that $q$ is absolutely continuous function excepted at $t_{\text {out }}$ where it exhibits a jump.

Taking these information into account we now get a analytical characterization of the solution and $q$ multiplier.

Step 1: $h(\bar{x}(t))<0$ for $\left.t \in] t_{\text {out }}, 12\right]$.

Since the inequality constraint is not active, then $p(t)=q(t)$. Thus we most have $p(12)=0$ and, since $\dot{p}(t)=\beta p(t)$, by the adjoint equation of the MP, we can conclude that $p(t)=q(t)=0$.

Applying the Weierstrass condition of MP, we get $\bar{u} \leq u, \forall u \in[0, M]$. Thus $\bar{u}=0$.

Replacing $\bar{u}$ by zero in the dynamics, we have:

$$
\dot{\bar{x}}(t)=g(t)-\beta \bar{x}(t) .
$$

As $\bar{x}\left(t_{\text {out }}\right)=x_{\text {min }}$, then $\bar{x}(t)=e^{-\beta\left(t-t_{\text {out }}\right)}\left(\int_{t_{\text {out }}}^{t} e^{\beta\left(s-t_{\text {out }}\right)} g(s) d s+x_{\min }\right)$, for $\left.t \in] t_{\text {out }}, 12\right]$. 
Therefore $(\bar{x}(t), \bar{u}(t))=\left(e^{-\beta\left(t-t_{\text {out }}\right)}\left(\int_{t_{\text {out }}}^{t} e^{\beta\left(s-t_{\text {out }}\right)} g(s) d s+x_{\min }\right), 0\right)$ and $q(t)=0$, for $\left.t \in] t_{\text {out }}, 12\right]$.

Step 2: $h(\bar{x}(t))=0$ for $t \in\left[t_{\text {in }}, t_{\text {out }}\right]$.

As for $t \in\left[t_{\text {in }}, t_{\text {out }}\right]: h(\bar{x}(t))=0$, we have $\bar{x}(t)=x_{\min }$. Therefore:

$$
\dot{\bar{x}}(t)=0 \Leftrightarrow \bar{u}(t)=-g(t)+\beta x_{m i n}
$$

And we may conclude that $(\bar{x}(t), \bar{u}(t))=\left(x_{\min },-g(t)+\beta x_{\min }\right)$ for $\left.t \in\right] t_{\text {in }}, t_{\text {out }}[$. Since $\bar{u}(t)>0$ for $t \in] t_{\text {in }}, t_{\text {out }}[$, then by the MP we also conclude that $q(t)=1, t \in] t_{\text {in }}, t_{\text {out }}[$.

Step 3: $h(\bar{x}(t))<0$ for $t \in\left[0, t_{\text {in }}[\right.$.

Since $h(\bar{x}(t))<0$, then $p(t)=q(t)$. Again, as $p\left(t_{i n}\right)=1$ and $\dot{p}(t)=\beta p(t)$, by the adjoint equation of the MP, we can conclude that $p(t)=q(t)=e^{\beta\left(t-t_{i n}\right)}$. On the other hand $p(t)=q(t)<1$, then by Weierstrass condition we get $\bar{u} \leq u, \forall u \in[0, M]$. Therefore $\bar{u}=0$.

Consequently, our dynamics is written as:

$$
\dot{\bar{x}}(t)=g(t)-\beta \bar{x}(t)
$$

Since $\bar{x}(0)=x_{0}$, then $\bar{x}(t)=e^{-\beta t}\left(\int_{0}^{t} g(s) d s+x_{0}\right)$, for $t \in\left[0, t_{i n}\right]$.

Therefore $(\bar{x}(t), \bar{u}(t))=\left(e^{-\beta t} \int_{0}^{t} g(s) d s+x_{0}, 0\right)$ and $q(t)=e^{\beta\left(t-t_{i n}\right)}$, for $t \in\left[0, t_{i n}\right]$.

Briefly,

$$
\begin{gathered}
\bar{x}(t)= \begin{cases}e^{-\beta t}\left(\int_{0}^{t} g(s) d s\right)+x_{0} & t \in\left[0, t_{\text {in }}\right] \\
x_{\min } & t \in] t_{\text {in }}, t_{\text {out }}[ \\
e^{-\beta t}\left(\int_{t_{\text {out }}}^{t} g(s) d s+e^{\beta t_{\text {out }}} x_{\min }\right) & t \in\left[t_{\text {out }}, 12\right]\end{cases} \\
\bar{u}(t)= \begin{cases}0 & t \in\left[0, t_{\text {in }}\right] \\
-g(t)+\beta x_{\min } & t \in] t_{\text {in }}, t_{\text {out }}[ \\
0 & t \in\left[t_{\text {out }}, 12\right]\end{cases} \\
q(t)= \begin{cases}e^{\beta\left(t-t_{\text {in }}\right)} & t \in\left[0, t_{\text {in }}\right] \\
1 & t \in] t_{\text {in }}, t_{\text {out }}[ \\
0 & t \in\left[t_{\text {out }}, 12\right]\end{cases}
\end{gathered}
$$


Next, we plot the numerical and analytical solution obtained from our model for the year 2010 .
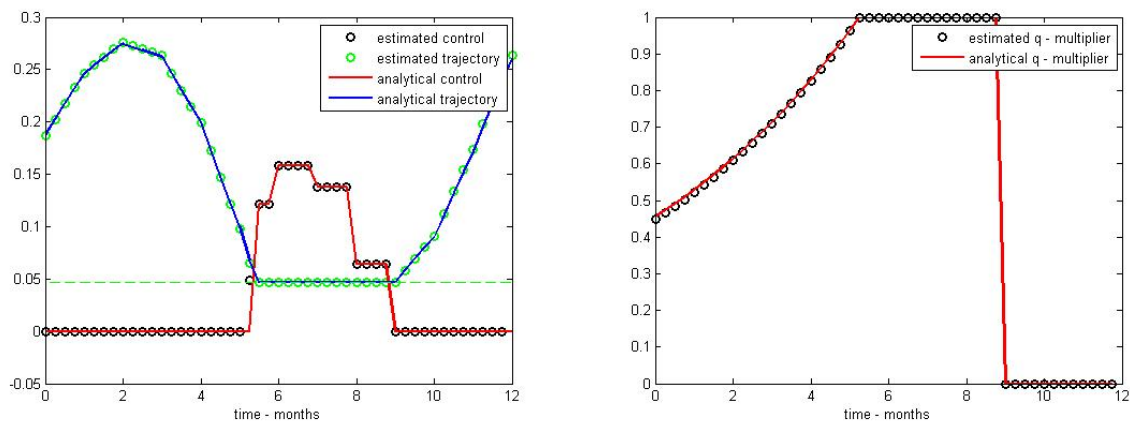

From this figure we confirm that the numerical solution agrees with the results shown in section 3 . We notice the analytical and estimated results of trajectory, control and multipliers coincide.

\section{Conclusion}

We characterized the optimal solution to the irrigation problem described as the minimization of the water flow (control) so that the amount flow of water in the soil (trajectory) fulfils the cultivation water requirements of the crop. We proved the existence of solution and we verify that the Maximum Principle conditions are also sufficient conditions. Furthermore, we characterized the solution applying the necessary conditions of optimality in the form of the Maximum Principle and we conclude that the multiplier $q(t) \leq 1$ when $\bar{u}=0$ and when $\bar{u} \in] 0,1[$ then $q(t)=1$. We used the information obtained in numerical solution with respect to the time interval where the state constraint is active to get the optimal solution analytically. Finally, we compare the results obtained analytically and numerically are compared showing conclude that the numerical solution fulfils the MP conditions.

\section{Appendix}

\section{Auxiliary results:}

Here, we present an adaptation of corollary of theorem 5.5.4 and theorem 5.4.2 from [2] to our problem, that can be written as: 


$$
\begin{aligned}
& (O P) \min \int_{0}^{T} L(x(t), u(t)) d t \\
& \text { subject to: } \\
& \dot{x}(t)=f(t, x(t), u(t)) \quad \text { a.e. } t \in[0, T] \\
& h(x(t)) \leq 0 \quad \forall t \in[0, T] \\
& u(t) \in \Omega \quad \text { a.e. } \\
& x(0) \in C_{0}
\end{aligned}
$$

Theorem 1. (Existence) (theorem 5.5.4 in [2]) Consider the (OP) problem and assume that $f$ and $L$ are differentiable in the state and control variables, $\Omega$ is convex bounded set, $h$ is lower continuous function in $x$ and $C_{0}$ is closed and convex and suppose in addition that:

i) For each $x$ with $h(x) \leq 0$, the following set is convex:

$$
\{[f(t, x, u), L(x, u)+\delta]: u \in[0, M] \text { and } \delta \geq 0\} .
$$

ii) There exist functions $\sigma(t), \rho(t)$ and $\phi(t, p)$ finite and summable in $t$ (with $\sigma$ and $\rho$ nonnegative) such that for all $x$ satisfying $h(x) \leq 0$ for all $w$ in $\Omega$ and for all $p$ one has

$$
H(t, x, p, w, 1) \leq \phi(t, p)+|x|(\rho(t)+\sigma(t))|p| .
$$

iii) The set $C_{0}$ is compact;

Then, if there is at least one admissible $(x, u)$ for $(O P)$ giving a finite value to the cost functional, there is a solution to $(O P)$.

Corollary 1. (Sufficient condition)(corollary of theorem 5.5.4 in [2]) Consider the $(O P)$ problem and assume that $f$ and $L$ are differentiable in the state and control variables, $\Omega$ is convex bounded set, $h$ is lower continuous function in $x$ and $C_{0}$ is closed and convex. Let $(x, u)$ be admissible for $(O P)$, and suppose that there is an arc $p$ satisfying:

i) For almost all $t$, the function $w \rightarrow H(t, x(t), p(t), w, 1)$ attains a maximum over $\Omega$ at $w=u(t)$.

ii) $-\dot{p}(t)=H_{x}(t, x(t), p(t), u(t), 1)$ a.e..

iii) $q(T)=0$.

iv) For each $t$, the function $(y, w) \rightarrow H(t, y, p(t), w, 1)-\psi_{\Omega}(w)$ is concave. $\left(\psi_{\Omega}(w)\right.$ is the indicator function.)

Then $(x, u)$ solves $(O P)$. 


\section{Acknowledgments}

The financial support of PEst-OE/MAT/UI0013/2014 and PEST-C/FIS/UI607/2013, European Union FP7 (FP7-PEOPLE-2010-ITN, Grant Agreement no. 264735SADCO), FCT projects PTDC/EEA-CRO/116014/2009 and PTDC/EEI-AUT/1450/2012 are gratefully acknowledged.

\section{References}

1. J. B. Betts. Pratical methods for optimal control using nonlinear programming. SIAM, Philadelphia, 1943.

2. F. H. Clarke. Optimization and Nonsmooth Analysis. Wiley-Interscience, New York, 1983.

3. S. Lopes F. A. C. C. Fontes. Normal forms of necessary conditions for dynamic optimization problems with pathwise inequality constraints. Journal of Mathematical Analysis and Applications, 399:27-37, 2013.

4. R. E. Horton. An approach toward a physical interpretation of infiltration capacity. Soil Sci. Soc. Am. Proc, 5:300-417, 1940.

5. R. Elliott D. Itenfisu P. Brown M. E. Jensen B. Mecham T. A. Howell R. L. Snyder S. Eching T. Spofford M. Hattendorf D. Martin R. H. Cuenca I. A. Walter, R. G. Allen and J. L. Wright. The ASCE standardized reference evapotranspiration equation. Rep. Task Com. on Standardized Reference Evapotranspiration, 2002.

6. L. S. Pereira. Necessidades de gua e mtodos de rega. Publicaes Europa - Amrica, 2004.

7. F. Rampazzo and R. B. Vinter. A theorem on the existence of neighbouring feasible trajectories with aplication to optimal control. 16:335-351, 1999.

8. J. R. Raposo. A REGA - dos primitivos regadios s modernas tcnicas de rega. Fundação Calouste Gulbenkian, 1996.

9. R. Pereira S. Lopes, F. Fontes and G. J. Machado. Irrigation planning in the context of climate change. Mathematical Models for Engineering Science-MMES11, pages 239-244, 2011.

10. R. Pereira M. Gonçalves S. Lopes, F. Fontes and G. J. Machado. Irrigation planning: an optimal control approach. International Conference of Numerical Analysis and Applied Mathematics, AIP Conference Proceedings, 1558:622-626, 2013.

11. R. Pereira M. Gonçalves S.Lopes, F. Fontes and G. J. Machado. An optimal control approach to the irrigation planning problem. Submitted to Conferncia Brasileira de Dinmica, Controle e Aplicaes, 2013.

12. R. Vinter. Optimal control. Birkhauser, Boston, 2000. 